\title{
Study to Cost of Air Spares Support Based on IPSO
}

\author{
Xiaohua Wang, Aiqin Mu, Fuhong Wang, Zhongbing Tang \\ The Fundamental Courses Department, Xuzhou Air Force College, Xuzhou, China \\ Email: \{plaxz, muaqin\}@126.com
}

Received October 11, 2011; revised November 9, 2011; accepted November 19, 2011

\begin{abstract}
Air spares support is general term of using and repairing of aircrafts which is the material foundation of aero technical support, its effectiveness influences operational effectiveness and equipments of aircrafts directly. Based on particle swarm optimization algorithm, a new model is proposed to optimize the distribution of the cost of air spares, it take the funds as resource and the improvement of performance efficiency as objective and deduces the expressions to get the best distribution plan. The results of experiments indicate that this model can make full use of the limited funds and obtain the highest efficiency of air spares support.
\end{abstract}

Keywords: Air Spares Support; Fund; Particle Swarm Optimization

\section{Introduction}

The aim of air spares support is supplying aircraft's flying and repairing with enough equipment. Its primary work is satisfying military's need of air spares immediately and exactly. There are several processes in air spares support, such as preparation, storage, supply and management [1], and all of these need sufficient funds. As reported in [2], the air force of USA spends about 0.8 billion to buy more than 500,000 consumptive materials annua$11 y$, and an F-15 flight squadron spends 31,000,000 dollars for its air spares support. It has being a major issue for how to improve the whole efficiency of air spares support thoroughly by making use of limit funds.

Many algorithms are using to improve the efficiency of air spares support. Reference [3] deduced expressions with the marginal analysis method, and obtained the scheme to get best effect cost of air spares support with the numerical calculate method. In [4], the sequential solution in dynamic programming is applied in solving the problem. This paper proposes a new model based on improved particle swarm optimization. In Section 2, the model of air spares support is constructed. Then an improved algorithm is presented in Section 3 to overcome local optimization of PSO. In Section 4, a simulated case is using to test the performance of new algorithm by comparing the results with other algorithm. Finally, conclusions are given in section 5 .

\section{A Model of the Cost of Air Spares Support}

In order to analysis the cost of air spares support quantitatively, several indicators are introduced. The support efficiency represents details that air spares support sys- tem has finished the goals of support task in a certain condition, which reflects the effect of system's support ability in the process of running it. It is synthetic calculation of the ability and military profits of air spares support [5]. The good rate of support is defined as follows.

$$
G=\frac{f}{Y} \times 100 \%
$$

in which, $f$ - the total days and numbers of aircrafts in good state, $Y$ - the total days and numbers of all aircrafts.

If the total days and numbers of all aircrafts in one airport is fixed, the good rate is generally in proportion to total days and numbers of aircrafts in good state, and it represents the ability of support. For these sakes, this paper take consider of total days and numbers of aircrafts in good state as effectiveness indicator of optimal model which presented as follows.

Assuming $W$ is the sum of funds of all support units, $x_{i}$ is the funds of support unit $i, Y_{i}$ is the total days and numbers of one aircraft supported by unit $i$ in airport, $G_{i}\left(x_{i}\right)$ is good rate, and $f_{i}$ is the total days and numbers of one aircraft in good state. Obviously, $f_{i}$ is function of funds $x_{i}$, the optimal distribution model of air spares support and be constructed as follows.

$$
\begin{gathered}
\max z=\sum_{i=1}^{n} f_{i}\left(x_{i}\right) \\
\text { s.t } \sum_{i=1}^{n} x_{i}=W \\
x_{i} \geq 0 \\
f_{i}=Y_{i} \times G_{i}\left(x_{i}\right)
\end{gathered}
$$


Because the good rate has consequentially regular law with the investment of funds, which means the more funds invested, the higher good rate will be reached. But the increment of good rate would decrease if funds invested over a number. It is obviously that the funds obey the standard normal distribution, and good rate is its distribution function, increment of good rate is its probability density, as in (6).

$$
G_{i}\left(x_{i}\right)=\int_{-\infty}^{x_{i}} \frac{1}{\sqrt{2 \pi} \sigma_{i}} \mathrm{e}^{-\frac{\left(t-\mu_{i}\right)^{2}}{2 \sigma_{i}^{2}}} \mathrm{~d} t
$$

in which, $\mu_{l}$-mean value of the $i$-th unit's funds, $\sigma_{i}$ mean square of the $i$-th unit's funds.

Although traditional algorithms such as dynamic programming can solve the model presented above, the process is inconvenient and times cost for computing increases with size $n$ and they have a positive negative exponential relation, the workload increases rapidly.

\section{Improved Particle Swarm Optimization}

PSO (particle swarm optimization) [6], a new intelligent optimization algorithm proposed by psychologist Kennedy and Dr. Eberhart in 1995, which intimates the bird swarm behaviors. It is a high efficiency algorithm and features many new functions and characteristics, such as concise conceptions, convenient for running, fast convergence and less parameters. At present, PSO is widely applied in many fields, such as functions optimization, training of neural network, fuzzy system control.

In PSO algorithm, each individual is called "particle", which represents a potential solution. When algorithm is running, all particles fly at random. Each of them keeps the best position "pbest" found by itself, besides, it also remembers all particles' best positions "gbest". Particles adjust their directions and velocities according two best positions found before.

In 1998, Shi proposed a new idea in [7], the weighting of inertia $w$ is introduced to improve PSO algorithm's performance. It is a perfect method and brings PSO algorithm to completion, which is called standard PSO algorithm now. Larger weighting of inertia will lead to high searching efficiency of global area of PSO, on the contrary, local area's efficiency will be more clearly. The renewed formulas of standard PSO are described as following.

$$
\begin{gathered}
v_{i}^{k+1}=w v_{i}^{k}+c_{1} r_{1}\left(p_{i}-x_{i}^{k}\right)+c_{2} r_{2}\left(p_{g}-x_{i}^{k}\right) \\
x_{i}^{k+1}=x_{i}^{k}+v_{i}^{k}
\end{gathered}
$$

in which, $x_{i}=\left(x_{i 1}, x_{i 2}, \cdots, x_{i D}\right)$ is the position of $i$-th particle in $D$-dimension; $v_{i}=\left(v_{i 1}, v_{i 2}, v_{i D}\right)$ is particle's velocity which represents its direction of searching; $i=1,2, \cdots$, $n, n$ is the number of particles; $k$ is iteration times; $r_{1}$ and $r_{2}$ are random values ranging between 0 and 1 , which are used to keep the diversity of particle swarm; $c_{1}$ and $c_{2}$ are learning coefficients, which are also called acceleration coefficients; $v_{i d}^{k}$ is the $d$-th component of velocity of particle $i$ in the $k$-th iteration; $x_{i d}^{k}$ is the $d$-th component of position of particle $i$ in the $k$-th iteration; $p_{i d}$ is the $d$-th component of the best position particle $i$ has ever found; $p_{g d}$ is the $d$-th component of the best position the particle swarm have ever found.

For avoiding being entrapped in local optimization, the idea of simulated annealing algorithm is used to improved PSO's performance. First, gets $p_{g}$ by running PSO one time, then runs simulated annealing algorithm in $p_{g}$ 's neighborhoods and calculates a new solution of $x$ '. Comparing the fitness of $p_{g}$ and $x$, if $x^{\prime}$ is better than $p_{g}$, runs PSO another time and renews $p_{g}$. Otherwise, a new value of $x$ ' is produced in $p_{g}$ 's neighborhoods based on simulated annealing algorithm. There is at most $L$ new solutions can be found, in which, $L$ is length of Markov chain in simulated annealing.

According to the problem in this paper, the way of generating a new value of $x^{\prime}$ is described as follows. First, assuming $p_{g}=\left(p_{g 1}, p_{g 2}, \cdots, p_{g n}\right), x^{\prime}=\left(x_{1}, x_{2}, \cdots, x_{\mathrm{n}}\right)$, two support units $i$ and $j$ are selected randomly, meanwhile, a random value of support funds is assigned to unit $i$, then setting $x_{\mathrm{i}}=p_{g i}-\triangle f, x_{\mathrm{j}}=p_{g i}+\triangle f$, and keeping the other units' funds invariable.

\section{A Case Study of Air Spares Support with IPSO}

Assuming an armed force has six units of air spares support, each of them supplies a flying regiment. According to statistical information in history, each unit's funds and total days and numbers of one year are shown in Table 1.

Using IPSO (improved particle swarm optimization) algorithm proposed in this paper, the parameters are setting as follows. The number of particles is 40 , the max iteration time is 500, learning factors $c_{1}$ and $c_{2}$ equal to 1.5 , initial weighting $w_{\max }$ is 1.5 , final weighting $w_{\min }$ is 0.6 , the max flying velocity $v_{\max }$ is 3 , the min flying velocity $v_{\min }$ is -3 , the length of Markov chain $L$ is 10 , and particle's position and velocity are initialized randomly.

In order to make it easy to apply the distribution scheme of air spares support in practice, this paper assumes that the funds allocate to each unit does not include decimal part. Program based on this algorithm is carried out in Matlab 50 times independently and the results are shown in Table 2.

From Table 2, it is shown that the maximum support days and numbers is 67,748 by using IPSO algorithm, and its good rate of support is $93.43 \%$. In [3], marginal analysis method is applied to solve the same problem and the result is 67,672 days and numbers of support, and the number of 67,697 is obtained in [4] by using dynamic 
Table 1. The funds and total days and numbers of each air spares support unit.

\begin{tabular}{|c|c|c|c|c|c|c|}
\hline & \multicolumn{6}{|c|}{ air spares support unit } \\
\hline & 1 & 2 & 3 & 4 & 5 & 6 \\
\hline $\begin{array}{l}\text { mean value of funds } \\
\text { (per ten thousands) }\end{array}$ & 67 & 67.3 & 59.4 & 71.8 & 71.2 & 62.8 \\
\hline mean square of funds & 4.4271 & 4.6306 & 6.3749 & 6.7052 & 6.8234 & 4.7074 \\
\hline $\begin{array}{l}\text { total days and numbers } \\
\text { of a year }\end{array}$ & 10,950 & 10,220 & 13,140 & 12,410 & 11,680 & 14,110 \\
\hline
\end{tabular}

Table 2. The running results based on IPSO.

\begin{tabular}{cc}
\hline the best distribution scheme & $74,74,69,81,81,71$ \\
\hline the maximum days and numbers of support & 67,748 \\
times of getting the best solution & 50 \\
the maximum iteration times & 87 \\
the minimum iteration times & 22 \\
average times of iteration & 53 \\
\hline
\end{tabular}

programming algorithm. It is clearly that using IPSO algorithm can support more days than using other algorithm proposed in [3,4], and IPSO algorithm can achieve the best solution every time. Furthermore, the maximum iteration times of this case is 86 and the minimum iteration times is 22 , it can get the best solution on average 53 times iteration, which indicates that IPSO algorithm has a feature of fast convergence velocity.

By running programs 50 times independently, the results of simulated annealing (SA), PSO and IPSO are compared in Table 3. It can be found that the best solution appears 16 and 41 times in experiments using SA algorithm and PSO, however, IPSO algorithm can get best solution every time. Furthermore, using PSO, it needs at least 242 times of iterations to get the best solution, but the maximum iteration times of IPSO is 100 only, which indicates new algorithm improved speed and rate of convergence.

\section{Conclusion}

It is easy to find an optimal distribution scheme on traditional optimize algorithm for air spares support, however, if the sum of funds is invariable, the performance of support can not achieve the best solution. As a new intelli-
Table 3. The comparion of using SA, PSO and IPSO.

\begin{tabular}{cccc}
\hline & SA & PSO & IPSO \\
\hline initial temperature & 10000 & $/$ & $/$ \\
ending temperature & 1 & $/$ & $/$ \\
annealing velocity & 0.87 & $/$ & $/$ \\
length of Markov chain & 10 & $/$ & 10 \\
max iteration times & $/$ & 1500 & 100 \\
the maximum days and numbers & 67,748 & 67,748 & 67,748 \\
the minimum days and numbers & 67203 & 64612 & 67,748 \\
average of days and numbers & $67,701.42$ & 67,066 & 67,748 \\
times of getting the best solution & 16 & 41 & 50 \\
\hline
\end{tabular}

gent optimization algorithm, IPSO algorithm can solve this problem successfully and features many other characteristics, such as fast convergence speed and very convenient for running, all of these implies that IPSO has a better performance in searching best solution of nonlinear programming, and it has some practical value in solve the optimization of large and medium-sized expense support problems.

\section{REFERENCES}

[1] X. C. Han, “The Air Spares Management," Blue Sky Press, Beijing, 2003.

[2] B. S. Blanchard "Logistics Engineering and Management," Prentice Hall, New York, 1998.

[3] R. C. Zhang and S. Z. Zhao, "Study to Optimize and Distribute the Cost of Air Spares Support," Journal of Beijing University of Aeronautics and Astronautics, Vol. 31, No. 1, 2005, pp. 102-104.

[4] F. Guo, C. Y. Liu and X. X. Guo, "Optimal Distribution of Aircraft-Spares Support Cost Based on Dynamic Programming Algorithm," Value Engineering, Vol. 25, No. 10, 2010, pp. 63-64.

[5] H. D. Zhu and Y. Zhong, "Evaluation of Air Materials Support Effectiveness Based on Utility Function," Logistics Technology, Vol. 26, No. 8, 2007, pp. 246-248.

[6] J. Kennedy and R. C. Eberhart, "Particle Swarm Optimization," IEEE International Conference on Neural Network, Perth, 1995, pp. 1942-1948. doi:10.1109/ICNN.1995.488968

[7] Y. Shi and R. C. Eberhart, "A Modified Particle Swarm Optimizer," Proceedings of Congress on Evolutionary Computation, Indianapolis, 4-9 May 1998, pp. 79-83. 